\title{
Probing Electron Beam - Nanoparticle Capping Ligand Interactions during Liquid Phase Transmission Electron Microscopy Using a Correlative Fluorescence Microscopy Assay
}

\author{
Thilini U. Dissanayake, ${ }^{1}$ Mei Wang, ${ }^{1}$ and Taylor J. Woehl ${ }^{1}$
}

${ }^{1}$ Department of Chemical and Biomolecular Engineering, University of Maryland, College Park, MD, USA.

Liquid phase transmission electron microscopy (LPTEM) has extensive applications in imaging dynamics of various nanoparticles (NPs) systems including self-assembly. The surface chemistry of NPs is changed with different capping ligands to manipulate the interparticle interactions between particles which is crucial in directing the assembly of NPs. Water - electron beam interactions during LPTEM produce different types of aqueous radicals and specially hydroxyl radicals are known to react rapidly with organic molecules $[1,2]$. Therefore, it is important to study the beam induced modifications to surface bound and free ligands of NPs to correctly interpret the results and minimize the interference during NPs assembly process in LPTEM.

Here fluorescence microscopy (FM) was utilized to image the effect of electron beam irradiation on surface bound and free capping ligands in LPTEM. We did dose controlled LPTEM imaging of a system of branched polyethyleneimine (BPEI) coated $100 \mathrm{~nm}$ silver NPs in DI water with scanning TEM (STEM) mode by changing dose rate $(0.144-0.616 \mathrm{MGy} / \mathrm{s})$ and irradiation time $(30 \mathrm{~s}-10 \mathrm{~min})$ at a magnification of $\times 20 \mathrm{k}$. Afterwards, BPEI ligands were fluorescently labelled by carboxy-flourescein which is specific to primary amine groups on the BPEI. Fluorescence intensity from FM is proportional to the local concentration of BPEI on SIN $_{x}$ membrane. FM revealed beam induced polymerization and degradation of BPEI ligands during LP-TEM imaging and there were two distinct features visible, bight regions and dark regions with bright halos (Figure $1 \mathrm{a}-\mathrm{c}$ ). The bright fluorescence corresponds to the regions irradiated for $<30$ seconds (Figure 1a) whereas regions irradiated for $>5$ minutes had darker or similar fluorescence compared to background (Figure $1 \mathrm{~b}, \mathrm{c}$ ). Hydroxyl radicals formed by electron beam irradiation produce BPEI radicals near the membrane and this observation of fluorescence changes could be associated to two competing polymer reactions of BPEI radicals [3]. The BPEI radicals undergo intermolecular crosslinking at $\mathrm{t}<30 \mathrm{~s}$ near the membrane, increasing local concentration of BPEI (high fluorescence intensity). At $t$ $>5$ mins chain scission overpowers crosslinking and scissored BPEI fragments are driven out of the image area by diffusion where they pile up at the perimeter giving bright halos.

The irradiated regions in an experiment without NPs where only free capping ligands were present is illustrated in Figure $1(\mathrm{e}-\mathrm{g})$. The regions irradiated for $<1$ min were barely discernible in contrast to bright fluorescence in the presence of NPs. As the time interval was increased to $5-10$ minutes, image areas showed increased fluorescence indicating crosslinking reactions between BPEI molecules when chain scission was prominent in the presence of NPs. This clearly indicate a disparity in dose between two cases and could be attributed to dose enhancement near a metal-water interface and by metal NPs [4, 5]. The kinetics were faster in the sample with NPs due to higher concentrations of radicals with increased local dose by silver NPs. When $1 \mathrm{M}$ tert-butanol was used as a radical scavenger in the system, it showed that tert-butanol slowed the overall reaction kinetics (Figure 2). The irradiated regions for $<1$ minute did not result in any fluorescence difference compared to the background, while image areas of $\mathrm{t}>5$ minutes showed slight increase of fluorescence compared to the background implying progression of 
intermolecular crosslinking [6, 7].

References:

[1] NM Schneider et al, J. Phys. Chem. C, 118, 38 (2014), p. 22373.

[2] TJ Woehl and P Abellan, J. Microscopy, 265(2) (2016), p. 135.

[3] M Matusiak, S Kadlubowski and JM Rosiak, Radiation Physics and Chemistry 169 (2020).

[4] T Gupta et al, Nanoscale 10 (2018), p. 7702.

[5] J Korpanty, LR Parent and NC Gianneschi, Nano letters, 21(2) (2021), p. 1141.

[6] TU Dissanayake, M Wang, TJ Woehl, ACS Appl. Mater. Interfaces (2021), 13, 31, 37553-37562

[7] The authors acknowledge funding from the Army Research Office (\#W911NF-20-1-0169).

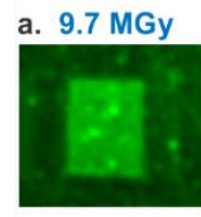

c. $194.1 \mathrm{Mgy}$

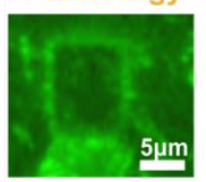

$5 \mu \mathrm{m}$ b. $97.1 \mathrm{Mgy}$

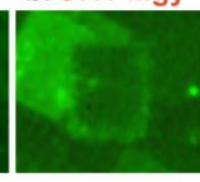

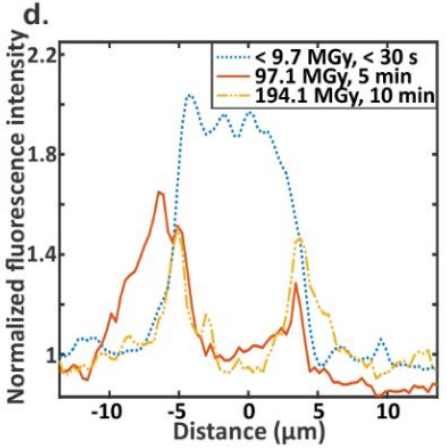
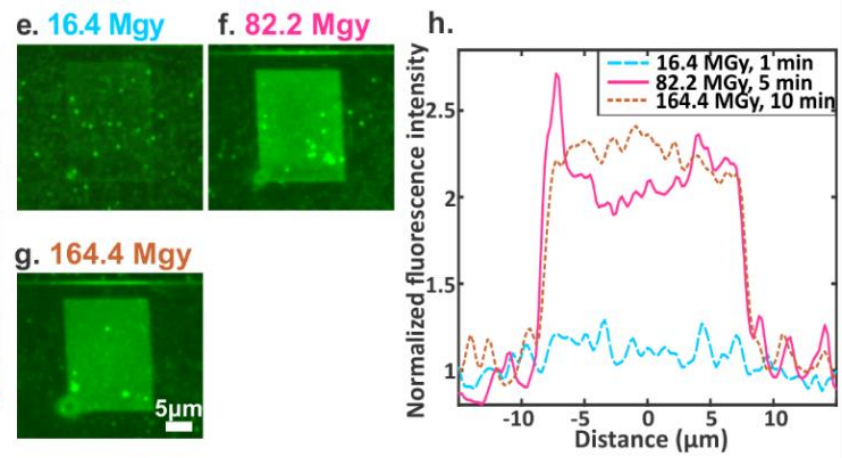

Figure 1. FM images $\left(\mathrm{a}-\mathrm{c}\right.$ ) are regions of the $\mathrm{SiN}_{\mathrm{X}}$ membrane irradiated at a dose rate of $0.324 \mathrm{MGy} / \mathrm{s}$ with nanoparticles in the cell (time: a. < $30 \mathrm{~s}$, b. 5 min, c. $10 \mathrm{~min}$ ). FM images (e - g) are regions of the SiN $x$ membrane irradiated at a dose rate of $0.274 \mathrm{MGy} / \mathrm{s}$ without NPs and only free ligand in the cell (time: a. $1 \mathrm{~min}$, b. $5 \mathrm{~min}$, c. $10 \mathrm{~min}$ ). (d) and (h) shows the fluorescence intensity along a horizontal line drawn across each irradiated region of the samples with and without NPs, respectively. Reprinted with permission from ref. 6. Copyright 2021 American Chemical Society.
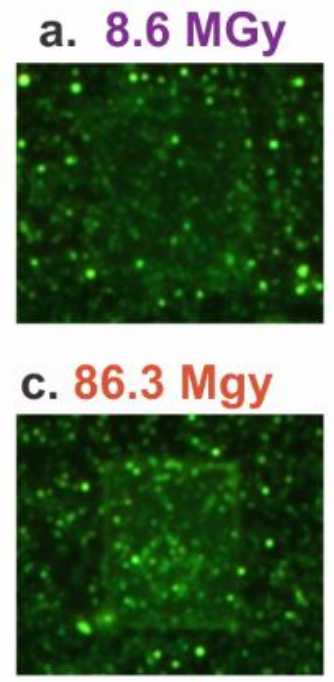

\section{b. $43.1 \mathrm{Mgy}$}
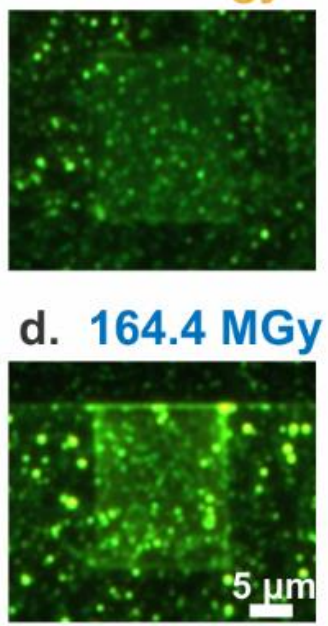

Figure 2. FM images of the silicon nitride membrane irradiated in the presence of tert-butanol. FM images corresponding to the regions irradiated at a dose rate of $0.144 \mathrm{MGy} / \mathrm{s}$ for times of (a) $1 \mathrm{~min}$ (b) $5 \mathrm{~min}$ and (c) $10 \mathrm{~min}$ and, at a dose rate of $0.274 \mathrm{MGy} / \mathrm{s}$ for times of (d) $10 \mathrm{~min}$. 